\title{
El fuerte de Santa Cruz y el conjunto defensivo español de Orán- Argelia
}

\section{Souad Metair}

Arquitecto, Orán, Argelia, souadoran@gmail.com

\begin{abstract}
The fort of Santa Cruz in Oran (Algeria) represents the peak of the iceberg of a very huge and developed defence system. Its strategic situation has been the key of the failure and the success of this system. In 1708 the Turkish army took control of Oran by mining the escarpment of Santa Cruz. When Spanish returned in 1732 their first goal was to secure the city by securing the fort of Santa Cruz. In this way the Spanish kings sent many famous engineers of that moment to develop Santa Cruz project thinking in a very original solution which was sculpting a gorge in the mountain to isolate the fort of the tableland, blocking this way any attempt to mine the fort. Our communication will treat those projects to analyse how Santa Cruz passed from being the weakness of the defence system to be its strongest point.
\end{abstract}

Keywords: Santa Cruz, Oran, Algeria, fort.

\section{Introducción}

La ciudad de Orán (Argelia) goza de uno de los sistemas defensivos más desarrollados del norte de África con más de veinte obras fortificadas, construidas por importantes ingenieros de la corona española entre el siglo XVI y el siglo XVIII. Este sistema defensivo resume tres siglos de presencia española en Orán, entre 1509 y1792, con un paréntesis otomanode1708a1732.

Erigido mayormente en el periodo de Felipe V, es contemporáneo de las grandes obras de los ingenieros italianos en Europa, ya sea en España, Italia o Flandes. En el caso de Orán, famosos ingenieros han sido responsables en el segundo periodo de la ocupación de casi todos los proyectos de la ciudad y de la imagen actual que tiene el conjunto defensivo en su paisaje urbano.

Las obras fortificadas de esta ciudad nunca han sido objeto de un estudio históricoarquitectónico, abarcando al fuerte de Santa Cruz como pieza clave para entender el conjunto defensivo, teniendo en cuenta que en tres siglos pasó de ser el punto débil del sistema, responsable de su caída en los manos de los turcos en 1708 a ser un fuerte inexpugnable en el segundo periodo. Esta evolución nos resultó de especial interés para estudiar.

En este sentido, la presente comunicación pretende analizar el fuerte de Santa Cruz como pieza destacable del conjunto defensivo español, apoyándose para ello en la investigación desarrollada durante el máster de conservación del patrimonio arquitectónico en forma de tesina de fin de máster.

La toma de Orán en 1509 fue parte del proyecto de ampliación de los dominios españoles en el norte de África para hacer frente a la piratería musulmana, que además de incomodar al comercio español en el mediterráneo, capturaba cristianos de Andalucía y del Levante para venderlos en tierras africanas. Frente a esta inseguridad, la toma de los presidios 
norteafricanos de Melilla hasta la Goleta, pasando por Orán y Mazalquivir, permitía proteger las tierras españolas desde Andalucía hasta Italia y al mismo tiempo dominar los reinos musulmanes del norte de África (Sánchez- Gijon, 2000)

Al llegar a Orán en 1509, los españoles se encontraron con varias obras fortificadas como la muralla árabe, Mazalquivir, Bordj el Ahmar y la Alcazaba (Lespes, 2003).Sin embargo, eran conscientes de la necesidad de mejorar y ampliar estas fortificaciones. En este periodo surge el proyecto de Santa Cruz en el punto más alto de la montaña del Murdjadjo frente a la meseta, cerrando de esta manera uno de los sistemas defensivos más estables del norte de África (De Epalza, 1988) y que les permitía protegerse de los continuos ataques de los turcos.

No obstante, la parte de la meseta que llegaba a Santa Cruz estaba totalmente desprotegida, lo que permitió a los turcos reconquistar la ciudad bombardeando el fuerte de Santa Cruz desde esta misma meseta, pero fue gracias a unas minas puestas en la parte oeste de su cortina, que finalmente lograron entrar abriendo una brecha, derrotando de esta manera a las tropas españolas Dada su posición alta, permitió además la capitulación del resto de las fortificaciones.

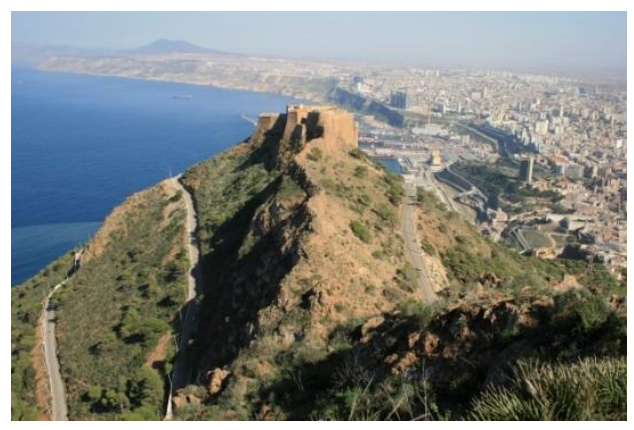

Fig. 1- Vista del fuerte de Santa Cruz desde la meseta (Souad Metair, 2013)

\section{La evolución constructiva de Santa Cruz durante la época española}

En la reconquista de Orán en 1732, todos los esfuerzos de los españoles se concentran en el fuerte de Santa Cruz, intentando ampliarlo con obras avanzadas $\mathrm{y}$, sobre todo pensando, una solución peculiar para fortalecer su punto débil frente a la meseta. En esta época la corona española envío algunos de sus mejores ingenieros para estudiar y dibujar nuevas propuestas para Santa Cruz.

El 18 de diciembre de 1732, casi seis meses después de la reconquista de Orán, el ingeniero Antonio Montaigu de la Pérille, reputado por sus realizaciones en Sicilia, Gibraltar y Cartagena, nos dejó un excelente plano en el que redibujó el fuerte anterior con todos sus detalles y señaló en color amarillo oscuro las obras que debían reconstruirse a partir de lo existente, es decir, lo que quedó derrumbado después del ataque de los turcos en 1708. De este modo, la obra más importante para reconstruir fue el espolón suroeste o, mejor dicho, el revellín que recibió el ataque y el bombardeo de los turcos desde la meseta y que se vio derrumbado casi por entero. Para proteger esta parte de la fortificación, el ingeniero dio prioridad a la creación de un foso con la elevación de un muro de tierra maciza que aguantaría los bombardeos desde la meseta.

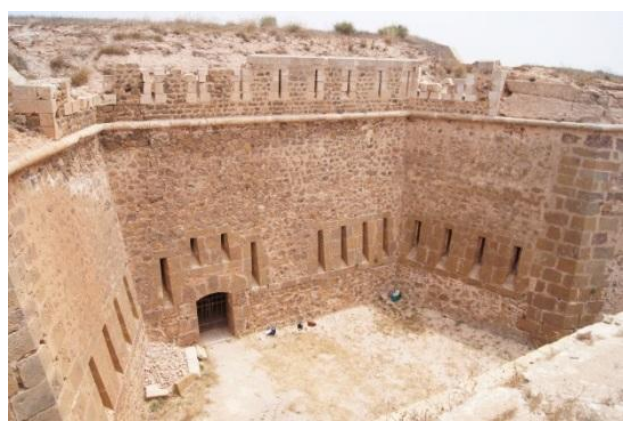

Fig. 2- Vista del foso suroeste desde el revellín (Souad Metair, 2013)

El ingeniero propuso en el mismo plano, reconstruir los cuarteles a prueba de bombas que podían recibir bombardeos desde la meseta, además de añadir baterías con espacios subterráneos a prueba de bombas que se encontraban justo después de la cortadura, protegidos con tierra maciza en su parte interior.

El ingeniero dio también gran importancia a la reconstrucción de la batería y los parapetos cercanos a los cuarteles de los oficiales. El muro que se encuentra después del foso y el revellín, frente a la meseta, se reconstruyó desde sus 
cimientos, además de la escarpa que propuso tallar alrededor de todo el fuerte con una medida de 30 pies de alto. Estas dos ejecuciones son las obras nuevas que recibiría esta fortificación el mismo año de la reconquista de Orán.

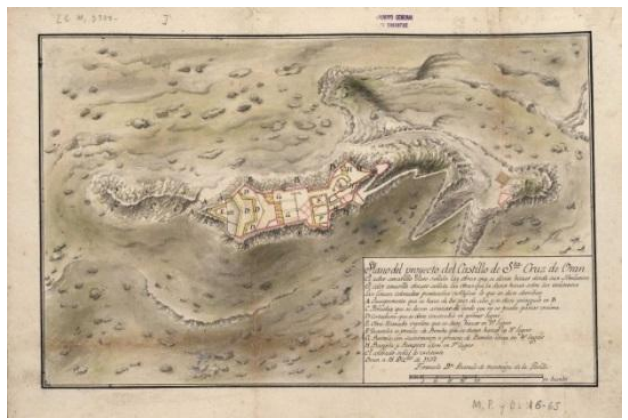

Fig. 3- Propuesta del ingeniero Antonio de Montaigu de la Pérille de 1732 (AGS)

Dos años más tarde, en 1734, se desarrolló otro proyecto llamado "Plano de una porción del castillo de Sta. Cruz, comprendidas las peñuelas", de autor desconocido, que revela que las obras en el fuerte estuvieron bastante avanzadas. Frente a la meseta, en la parte suroeste, distinguimos que el espolón o el revellín que fue volado durante el ataque de los turcos en 1708, ya estaba reconstruido en esta fecha, con dos plantas y las bóvedas que le convenía. Además del revellín, el foso que lo separa del fuerte fue ejecutado y se les añadió unas caponeras en la parte sur. Las escarpas del fuerte también habían sido ejecutadas. La cortadura, que debía haber sido ejecutada en la roca que unía el fuerte a la meseta, como ya había propuesto Antonio de Montaigu de la Pérille en 1732, estaba sin acabar en la fecha del plano.

Podemos observar también que se añadió un reducto con dos bóvedas debajo del revellín que sufrió la brecha con unas escaleras para acceder desde el foso ejecutado, así como un nuevo hornabeque con cuatro bóvedas bajo su terraplén y las baterías ganaron más capacidad de fuego. No obstante, en esta etapa de la reconstrucción de Santa Cruz, se había empezado la cortadura que debía separar y aislar el fuerte de la meseta, una cortadura sobre la que se profundizaría más adelante, durante el siglo XVIII.

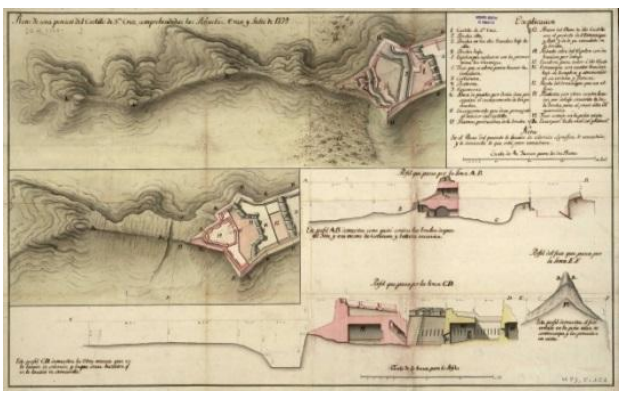

Fig. 4- Plan de 1734 de autor desconocido (AGS)

Transcurridos dos años, En el plano del 26 de septiembre de 1736 del ingeniero Juan Ballester y Zafra, podemos ver con más detalle las obras ejecutadas y todas las modificaciones aportadas por el autor. En primer lugar, el revellín frente a la meseta quedó igual con dos bóvedas en su parte inferior. Pasando el foso, que fue proyectado en 1732, nos encontramos con el primer frente con seis bóvedas bajo su terraplén y una escalera de caracol. Si se observa bien, se aprecia que este frente quedó igual que lo que había proyectado anteriormente Antonio Montaigu de la Pérille. Sin embargo, en la batería que aparece justo detrás, se le adjuntó dos caponeras, una a su derecha y otra a su izquierda.

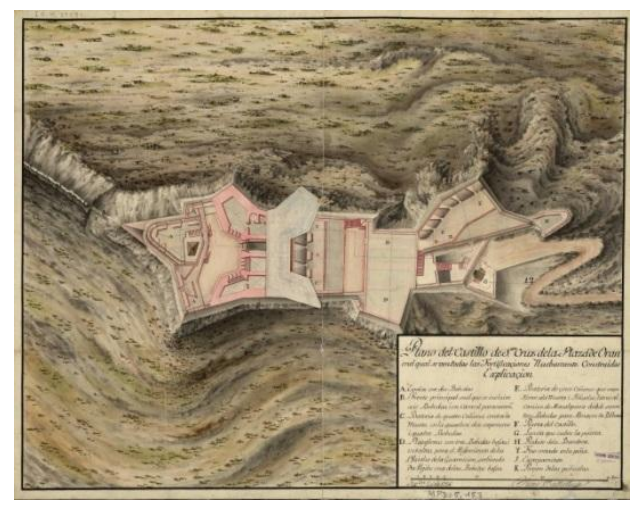

Fig. 5- Propuesta del ingeniero Juan Ballester y Zafra de 1736 (AGS)

El 11 de febrero de 1771 en el dibujo titulado "Plano y perfil en que se manifiesta el estado actual de la cortadura de la meseta", notamos que todavía el corte que separa la meseta de Santa Cruz no se había terminado del todo desde la reconquista de Orán en 1732. El ingeniero 
Miguel González Dáviles explicó que el corte se ejecutó haciendo explotar artillería que hizo levantar la roca lo que hacía más fácil tallarla. En esta fecha la cortadura era de 7 varas, es decir, alrededor de 5,84 metros de profundidad y, 25 varas, es decir, 20,87 metros de largo. Esta cortadura fue $\tan$ fina que, en condiciones normales, una persona no puede cruzarla de pie. Desde entonces, Santa Cruz pasó a ser una fortificación inexpugnable del lado de la meseta y ningún minero se atrevía a pasar la cortadura para abrir una brecha como la de 1708 .

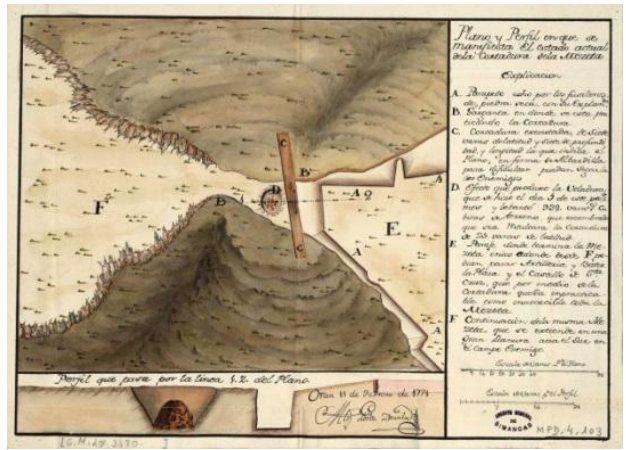

Fig. 6- Propuesta del ingeniero Miguel González Dávilas de 1771 (AGS)

\section{La evolución constructiva de Santa Cruz durante la época francesa}

En la época francesas, se procedió a la total restauración del fuerte de santa Cruz, según el informe titulado "Projets pour fortifications 1854-55", redactado por M. Curtet, teniente coronel-jefe de los ingenieros militares, y $\mathrm{M}$. Farre, capitán de los ingenieros militares, podemos deducir que el proyecto de restauración de Santa Cruz de 1854 se basó en su mayoría en un proyecto anterior redactado por el coronel Bodson de Noirfontaine en 1851. Sin embargo, algunas obras de restauración para adaptar la fortificación al uso de la guarnición francesa fueron iniciadas desde principios de 1853. El comandante superior decidió que era urgente hacerlos para permitir la defensa adecuada de la ciudad de Orán y del puerto de Mers-El-Kébir (Mazalquivir para los españoles), ya que es un punto estratégico en la defensa de la ciudad, que fue utilizado anteriormente por los turcos para atacar a los españoles.
El proyecto inicial de Badson de Noirfontaine de 1851 consistía principalmente en la restauración de la fortificación tal como existía después de su reconstrucción completa en el segundo periodo español, ya que partes de la fortificación habían sido destruidas por los españoles antes de dejar la ciudad en manos de los turcos, como estaba previsto en el acuerdo de 1792 con el Dey gobernador de Argelia.

Con los fondos de 1855 se pudo reconstruir el revellín frente a la meseta y una plaza de armas fue excavada hasta el nivel del foso. Además, se restauró la escarpa frente a la meseta del hornabeque: se reconstruyeron los muros de la cortina con sus aspillas, se terminó la restauración de la gran casamata con su bastión noroeste, se reconstruyó la bóveda y se reparó la cisterna del fuerte. La cortina sur que va desde el hornabeque oeste hasta el bastión este se reconstruyó desde sus cimientos.

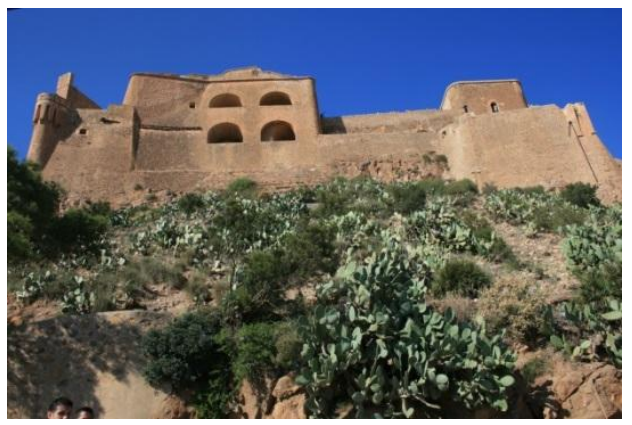

Fig. 7- Vista de la cortina sur (Souad Metair, 2013)

Esta cortina fue reconstruida desde sus cimientos por el mal estado en el que se encontraba su albañilería. En el proyecto inicial el trazado de esta cortina iba a ser modificado pero, como ya estaba la escarpa apoyada en la roca de la montaña, era lógico que la cortina siguiera su trazado. Los franceses mejoraron también el sistema de recuperación de aguas de la lluvia con unas tuberías de fundición desde las terrazas al aljibe.

Según nuestra observación, esta restauración determina el trazado final de la fortificación actual tal como la conocemos. Se reconstruyeron varios paramentos de nuevo, ya que estaban totalmente destruidos, lo que permitió cambiar 
el trazado en algunas ocasiones y otras veces seguir el trazado de los cimientos existentes. Se pidieron posteriormente más fondos para continuar con la restauración de Santa Cruz entre 1856 y 1857 , complementando la cortina del frente sur y su paralelo del frente norte, igual que la plaza de armas del revellín frente a la meseta. Del mismo modo se restauró el baluarte suroeste del hornabeque y su cortina.

\section{Conclusiones}

En el segundo periodo español y gracias a los archivos analizados podremos concluir que el nuevo trazado de la fortificación empieza a adoptar elementos de la fortificación abaluartada, introducidos por tratadistas franceses como Vauban o Pagan, etc. Estos elementos se concretan en las figuras del revellín o el reducto como obras avanzadas. Además, podemos observar que los baluartes del fuerte no siguen un trazado perfecto como exige la teoría, sino que se originan a partir de la topografía del terreno, aplicando de esta manera una de las pautas del Mariscal de Vauban que estipula que una fortificación no tiene por qué seguir un patrón preestablecido, sino que debe tener una solución única para un lugar único, es decir, que cada fortificación debe adaptarse al sitio que le rodea(Wezler, 2000), presentando obras avanzadas frente a los sitios más probables de ser atacados, que, en el caso concreto de Santa Cruz, es la parte suroeste frente a la meseta.

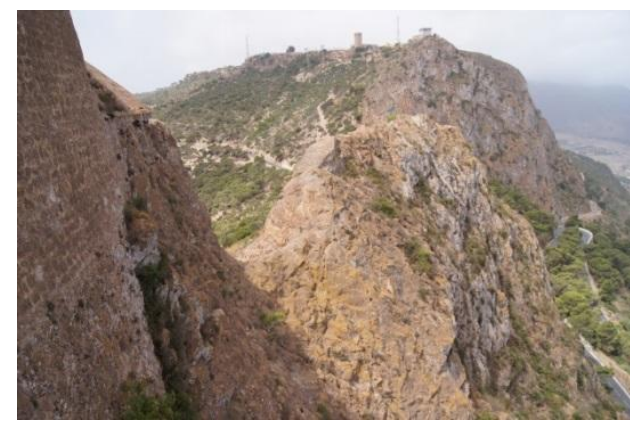

Fig. 8- Vista de la cortadura desde el revellín (Souad Metair, 2013)

En 1771 Se cerró el paso frente a cualquier minero que quisiera acceder desde la meseta, ya que la cortadura era tan fina y tan profunda que una persona para cruzarla en condiciones normales no podía hacerlo de pie sin caerse, lo que hacía que en condiciones de guerra fuera imposible contemplar esta posibilidad. Esta cortadura, que impide cualquier paso o aproximación hacia Santa Cruz, fue la solución idónea y ejemplar decidida por los ingenieros españoles, lo que permitió que Santa Cruz pasara de ser el punto débil de este conjunto defensivo a ser una fortificación inexpugnable, de forma que el guión de 1708 no volviera a repetirse.

\section{Referencias}

De Epalza M. Vilar J.B. (1988) Planos y mapas hispánicos de Argelia siglos XVI-XVIII. Plans et cartes hispaniques de l'algérie XVI-XVIII siècles. Instituto Hispano-Árabe de Cultura. Madrid. p. 102.

Lespes R. (2003).Études de géographie et d'histoire urbaine. Ed. Bel Horizon. Oran. p. 64.

Sánchez Doncel G. (1991) Presencia de España en Orán (1509-1792). Toledo. p. 420.

Sánchez- Gijon A. (2000) "La Goleta, Bona, Bugía y África, los presidios del reino de Túnez en la política mediterránea del emperador" in Las fortificaciones de Carlos V. Del Umbral. AEAC. Ministerio de Defensa. Sociedad Estatal para la conmemoración de los centenarios de Felipe II y Carlos V. Madrid. pp. 625- 626.

Wezler C. (2000) Architecture du bastion, l'art de Vauban Collection Architecture. Ouest-France. p. 18. 
